\title{
Reflexión acerca de la formación de ingenieros y la educación energética

\section{Resumen}

En este artículo se presenta el proyecto de investigación titulado "Factores que inciden en los procesos de enseñanza sobre el concepto de energía que favorecerían la educación de ingenieros en el marco de la sostenibilidad". Se realiza una breve exposición de algunos antecedentes, el problema planteado una primera aproximación al marco teórico y la metodología que se propone que permita dar cuanta de los objetivos del proyecto.

\section{Abstract}

In this work one presents the development of a project of research, which seeks to establish factors that affect in the processes of education on the concept of energy that they would favor the engineers education in the frame of the sustainability. For this intention one works with teachers of the university Institution the liberators with whom it is investigated by the way in how they teach and spread the concepts of energy and his relation with the sustainability in the context of the energetic education.

\section{Palabras clave:}

Educación energética, sostenibilidad, formación de ingenieros.

\section{Key words:}

Energetic Education, Sustainability, Engineers Formation

1 Estudiante del Doctorado en Educación de la Universidad Distrital Francisco José de Caldas. Bogotá, D. C. E-mail: japata25@gmail. com.

2 Estudiante del Doctorado Interinstitucional en Educación, Universidad Distrital Francisco José de Caldas, Bogotá, D. C. E-mail: jecm1419@yahoo.com.

3 Profesor de la Fundación Universitaria Los Libertadores y Profesor de la Universidad Antonio Nariño. E-mail: jecastrom@udistrital. edu.co. 


\section{Introducción}

Frente al problema energético que se presenta en la actualidad relacionado con problemas como el calentamiento global y el cambio climático, se ha hecho necesario aportar al desarrollo de la educación sostenible de los ciudadanos.

Un protagonista social, que posee una gran responsabilidad en el manejo y cuidado de los recursos energéticos, es el ingeniero, más aun cuando recaen sobre él procesos de transformación que deben ser más eficientes y que se ven obligados estar permeados por parámetros de cuidado y preservación del medio ambiente. Ésta responsabilidad del ingeniero se configura en tanto que su formación en ciencias básicas sea óptima, y cuando se relacionan con conceptos como el de energía.

La problemática sobre las fuentes de energía se caracteriza por dos aspectos fundamentales: a) el agotamiento de las fuentes de energía de origen fósil y b) la incidencia negativa de este tipo de recursos sobre el ecosistema terrestre, se ha venido constituyendo como un nuevo campo de conocimiento que tiene implicaciones como: el replanteamiento de la relación del ser humano con en el medio ambiente, una revaloración del experimento a partir de la simulación computacional y la necesidad de una mirada compleja que requiere de la interdisciplinariedad; en este nuevo campo de conocimiento uno de los fenómenos en los que se centra la atención es el cambio climático y en particular el calentamiento global (Flannery, 2006; Weart,.2006; Lovelock, 2006; y Brown, 2008). La fuerte incidencia que tienen fenómenos como el cambio climático y su relación estrecha con el uso y abuso de la fuentes de energía, sobre la vida en el planeta, requieren acciones desde la educación en ciencias donde se busque formar ciudadanos que logren una buena compresión del discurso científico emergente, por ejemplo, se hace importante que se realicen procesos de reconceptualización de conceptos como el de energía.

Los profesores de ciencias y particularmente los de física poseen una responsabilidad grande en los procesos de enseñanza en conceptos como el de energía. El discurso y las acciones del profesor influye lo que aprenden los estudiantes, por lo tanto se debe procurar que tanto discurso como acciones sean lo más adecuadas posibles. Lo que el profesor hace en clase está influenciado por sus vivencias escolares como estudiante y profesional como profesor en ejercicio, y especialmente por los libros que le sirvan de guía para desarrollar sus clases, muchos de los desarrollos presentados en el aula de clase poseen la forma y los argumentos presentados en los libros de texto.

Desde diversas investigaciones en la didáctica de las ciencias naturales, cuando se analizan lo que dicen los libros de texto, lo que saben profesores y estudiantes, se han identificado algunas dificultades en los procesos de enseñanza y aprendizaje del concepto de energía, algunas de estas son: polémica sobre la manera de introducir el concepto de trabajo antes que el de energía o viceversa (Sexl, 1981; Duit, 1981; Warren, 1982), dificultades en la interpretación de trabajo y esfuerzo (Driver y Warrington, 1985), interpretar inadecuadamente el concepto de trabajo, sin relacionarlo con trabajo y energía. (Duit, 1984), asociar la energía con alguna forma material (Duit, 1987; Solomon, 1985), tener la concepción que la energía puede gastarse o almacenarse (Solomon, 1985), no diferenciar entre tipos y fuentes de energía (Carr y Kirkwood, 1988; Solomon, 1985), atribuir la energía potencial al cuerpo y no a la interacción existente entre los cuerpos de un sistema de referencia (Solbes y Martín, 1991), no tener conocimiento sobre la variación de la energía interna (van Huls y van den Berg, 1993), considerara el calor como una forma de energía y atribuirle propiedades de sustancia (Albert, 1978; Erickson, 1979, 1980; von Roon, van Sparng y Verdonk, 1994), no diferenciar claramente los conceptos de calor y temperatura (Arnold,1994), y no interrelacionar los esquemas de transformación conservación, transferencia y degradación de la energía. (Duit, 1981, 1984). Estas dificultades deben tenerse en cuenta en los diversos momentos que caracterizan un trabajo de aula, estos son: planeación, gestión y evaluación. En el momento de planeación el profesor diseña un plan de trabajo que sirve como elemento orientador para llevar a cabo las actividades dentro de 
su clase, en este documento deben reposar sus intensiones, los contenidos y la metodología a trabajar. Para el caso particular del concepto de energía, como concepto fundamental de la física, debe hacerse referencia en todos y cada uno de los planes de trabajo desde el primer curso hasta el último.

Investigaciones más recientes abordaron otras problemáticas relacionadas con la enseñanza del concepto de energía, para indagar cómo los libros de texto y los profesores, de educación preparatoria en España, trabajan las estructuras y posturas relacionadas con la manera en cómo se introducen los esquemas y conceptos inherentes al tema de la energía tales como: hasta qué punto se tiene en cuenta en la enseñanza las ideas previas de los estudiantes, sus dificultades, y la necesidad de activar los esquemas de transformación conservación, transferencia y degradación de la energía; realización de un análisis crítico de la ley de conservación clasificando si se trata de teoremas o de principios; como se muestra el principio de conservación de la energía como un principio, no solo de la mecánica clásica sino de toda la física (Solbes y Tarín,1998)

A pesar de poder comprobar la existencia de resultados de investigación como los anteriores, y en términos generales los emanados desde la didácticas de las ciencias, tiene una incidencia en la formación de profesores de ciencias bastante modesta a pesar de llevar algo más de cuatro décadas, más modesta puede resultar su incidencia en procesos de formación de ingenieros (Carmona, A. 2009; Guisasola, J., Gras, A., Martinez, J. Almudi, J., Becerra, C. 2004). La compresión del concepto de energía por parte de ingenieros se hace necesario en tanto que su profesión implica el diseño, construcción e implementación de artefactos y procedimientos tecnológicos que redundan en la búsqueda de mayor eficiencia energética, reduciendo procesos de degradación, optimizando procesos de transferencia y transformación de energía, y comprendiendo como principio fundamental de la naturaleza la conservación de la energía. Las decisiones que deben tomar los ingenieros sobre temas energéticos, se supone, están mediadas por la compresión que estos tengan sobre el concepto de energía, cuyas bases en gran parte debieron haberse forjado en los procesos de enseñanza y aprendizaje que vivenciaron cuando eran estudiantes de ingeniera. No se puede dejar de lado el hecho que la incidencia de las decisiones tomadas por los ingenieros en los procesos tecnológicos, es de vital importancia en el cuidado del ecosistema terrestre, es decir que más que procurar el desarrollo de la civilización debe apuntarse al cuidado y preservación de la vida en el planeta, en otras palabras lo que se debe procurar es un desarrollo sustentable y sostenible.

Desde esta perspectiva, en este artículo se muestra el trabajo que se está adelantando, en la Fundación Universitaria Los Libertadores, dentro de la investigación titulada: factores que inciden en los procesos de enseñanza sobre el concepto de energía que favorecerían la educación de ingenieros en el marco de la sostenibilidad. Esta busca indagar por el estado actual de la educación energética en los procesos de formación de los ahora estudiantes de ingeniería de la Fundación, con el objetivo de generar instrumentos de evaluación y soporte que permitan replantear las actuales lineamientos educativos y didácticos, en busca de generar una conciencia ambiental y de sostenibilidad para con el planeta, desde la visión tecnológica y empresarial del quehacer diario de la ingeniería.

\section{Formulación del problema}

La situación actual de emergencia planetaria hace pensar en la necesidad de cambio en las concepciones ambientales, ecológicas y energéticas que requiere la sociedad, esta manera de pensar debe verse reflejada en los procederes individuales y colectivos de la industria, la ciencia y la educación. Teniendo presente esta problemática global y la atención a la situación del mundo en el manejo de la energía, se deben emprender diversas medidas orientadas a mantener o mejorar las condiciones de sustentabilidad de la tierra. La tenue y exponencial voz de alerta que emerge desde la transformada naturaleza del planeta, junto con las políticas ambiéntales y de educación que promueven algunas organizaciones mundiales, como la Organización de Naciones Unidas (ONU) y la Organización de los Estados Americanos (OEA), representan la base para 
generar estructuras y estrategias de cambio en los procesos educativos mundiales de todos los niveles de la educación. Como una de las estrategias de innovación educativa se presenta el llamamiento de la Naciones Unidas para que toda la educación contribuya a formar una ciudadanía consciente de los problemas a los que la humanidad ha de hacer frente y preparada para la necesaria toma de decisiones (Alcantud, 2007).

La problemática existente de las fuentes de energía como el calentamiento global, el agotamiento de las fuentes energéticas de origen fósil, entre otros, ha originado el desarrollo de políticas orientadas a la disminución del impacto ambiental, subrayando que la educación es un elemento indispensable para alcanzar el desarrollo sostenible. En referencia a estas estrategias la Organización de las Naciones Unidas para la Educación, la Ciencia y la Cultura (UNESCO) designo proclamar el período de diez años, entre el 2005 y el 2014, como la década de las Naciones Unidas para la Educación por el Desarrollo Sostenible. En donde se pretende promocionar la presentación de proyectos y recomendaciones a los gobiernos sobre la forma de promover y mejorar la integración de la educación para el desarrollo sostenible en sus respectivos planes de acción y estrategias de los sistemas educativos, invitando a todos los países a que lleven a cabo esfuerzos para ampliar la cobertura de su educación y que sus ciudadanos aprendan los valores, comportamientos y estilos de vida adecuados para un desarrollo sostenible (Década por una Educación para la Sostenibilidad, 2002).

Se debe plantear, entonces, la pertinencia de investigaciones relacionadas con la enseñanza/aprendizaje de la energía, que es uno de los conceptos básicos que contempla el currículo de la educación científica. La educación energética, es una problemática en la didáctica de las ciencias que actualmente está emergiendo como susceptible de investigación, debido a las dificultades de aprendizaje detectadas, (Solbes y Tarín, 1998; y Arias, 2006), y a la necesidad de construir un presente sustentable y un futuro sostenible. De esta manera, la realización de trabajos que involucren estudios de cómo se trabaja aborda actualmente la enseñanza de la energía, desde referentes de mayor amplitud como por ejemplo, el enfoque Ciencia, Tecnología, Sociedad y Ambiente (CTSA), podría dar cuenta de la problemática existente sobre las concepciones energéticas del contexto y procedimientos presentes en los estudiantes de las áreas relacionadas con las ciencias y la ingeniería. La ambiciosa y ardua tarea de ésta denominada "educación energética" se enmarca en replantear la manera de cómo se concibe la energía desde su contextualización en el aula hasta su aplicación y manejo en el desarrollo mundial, estudiando y evaluando todo el marco global inherente al tema como las fuentes energéticas renovables y no renovables, disponibilidad de recursos, eficiencia energética, el desarrollo industrial y el impacto ambiental.

La pertinencia de abordar el tema relacionado con la educación energética, se enmarca en el hecho que la problemática ambiental a nivel mundial es un parámetro que se debe tener en cuenta en cualquier tipo de implementación y avance científico e industrial, en lo que se denomina el uso de las tecnologías limpias como lo son: energía solar, energía eólica, biocombustibles, medios de transporte, energía móvil, edificios verdes, redes inteligentes y tratamiento de agua (Pernick \& Clint, 2000). Todos estos desarrollos tecnológicos evidencian claramente el papel fundamental que los ingenieros juegan dentro de los procesos de desarrollo industrial, económico y social de la humanidad. De esta forma el realizar una investigación como la que se propone en este trabajo permitirá iniciar procesos al interior de la institución que propendan por un cambio de la manera en cómo se forman los ingenieros dentro de la Fundación Universitaria Los Libertadores. Lo cual les posibilitará desarrollarse profesionalmente con una visión más amplia y contemporánea en el manejo energético y ambiental, que son los pilares fundamentales de cualquier tipo de desarrollo industrial. Así la realización de la investigación pretende desarrollar esquemas integrales en donde el ingeniero, además de su saber en la disciplina, adquiera formación en valores ecológicos, concepciones y actitudes hacia el medio ambiente, que le servirán posteriormente para poder ser partícipe de la transformación de procesos industriales, en busca de mejorar la eficiencia 
energética y disminuir el impacto ambiental a través de la generación de cambios en los paradigmas tecnológicos y científicos.

Teniendo en cuenta la situación anteriormente expuesta, el problema que se está trabajando se puede enunciar con la siguiente pregunta: ¿Qué factores favorecerían el entendimiento y el aprendizaje sostenible del Ingeniero a partir de la enseñanza del concepto de energía y sus procesos industriales y tecnológicos? Para poder contestar esta pregunta se deben dar respuesta a las siguientes preguntas subsidiarias: ¿Cómo se están planteando los procesos de enseñanza del concepto de energía para la formación de ingenieros desde el departamento de ciencias básicas de la Fundación Universitaria Los Libertadores?, ¿Cuál es la actitud que tienen los profesores de física para ingenieros sobre los procesos de enseñanza del concepto de energía del departamento de ciencias básicas de la Fundación Universitaria Los Libertadores?.

\section{Aproximación a la construcción del marco teórico}

En principio se reconoce que hablar de energía, va más allá de un problema conceptual, es una temática de las ciencias naturales que requiere un tratamiento especial ya que es un elemento fundamental en la atención a un problema como el energético. Es así, que se hace pertinente, en primer lugar, realizar inicialmente una reconstrucción histórica del concepto de energía que permita visibilizar su carácter dinámico y los referentes sociales, culturales y económicos que le circundaron. En segundo lugar, se presenta la lógica en la que se debe circunscribir el tratamiento del tema de la energía, esto es la sostenibilidad y términos más particulares el desarrollo sostenible. En tercer lugar se inicia una reflexión sobre las acciones educativas realizadas en América latina para hacer frente al problema energético. En cuarto y último lugar se perfila una propuesta educativa que podría hacer frente de manera más clara y contundente desde el ámbito educativo al problema energético, esto es, la educación energética, donde se ve este tema en términos de contenidos más allá de lo conceptual recono- ciendo también, los contenidos procedimentales y los axiológicos.

\section{Algunos elementos históricos del concepto energía}

Por otro lado, para analizar las dificultades en la enseñanza de la energía se debe tener en cuenta que la elaboración del concepto de energía fue un proceso que tardo más de dos siglos para llegar a su total comprensión con el principio de conservación de la energía. Este desarrollo epistemológico se llevó a cabo a través de dos etapas iníciales: como una primera aproximación se realizaron algunos intentos por establecer el principio de conservación de la energía, enmarcado dentro del estudio de los choques elásticos, con las imprecisiones que solamente se tenía en cuenta la energía cinética. Medio siglos más tarde Lagrange (1788) en su mecánica analítica, establece la conservación de la energía para un sistema mecánico. En la segunda etapa, físicos reconocidos como Joule y Helmholtz (1847) introducen la extensión de este principio, como un componente de toda la física, en el campo de la termodinámica donde los procesos de conversión de energía, transformación de calor en trabajo y el estudio de máquinas térmicas dan lugar a la formulación de los dos principios de la termodinámica.

Avances posteriores en el conocimiento de la física permitieron conceptualizar de manera más clara el comportamiento isotrópico del universo y la interpretación física sobre el comportamiento de la naturaleza. Con la aparición de la teoría electromagnética se establecen procesos de transferencia de energía y conservación de la energía, entre los campos y la radiación, relacionando la variación en la energía de los campos en una región del espacio con la radiación y el trabajo realizado por los campos sobre las cargas y corrientes inmersas en esta región. Interpretaciones mucho más sofisticadas en la denominada física moderna y la posterior mecánica cuántica dan cuenta de la universalidad del principio de conservación de la energía, cuando se relacionan la equivalencia de la masa y de la energía, y la energía de la masa en reposo o la relación de Heisenberg para energía-tiempo con la 
cuantificación de la energía y su indeterminación. Todas estas confirmaciones sectoriales a lo largo de la historia a cerca de la construcción y afirmación del principio de conservación de la energía, son enmarcadas de manera global por Noether quien plantea con una óptica más profunda la conservación de la energía al relacionarla con una invarianza de las leyes naturales bajo las traslaciones temporales.

\section{Medidas tomadas en América latina}

Reformas educativas que se han venido labrando en los nuevos currículos latinoamericanos iniciando el siglo xxI, muestran que los objetivos son ambiciosos pues pretenden replantear los sistemas educativos centrando la formación integral en el desarrollo de competencias y habilidades para la competitividad y el actuar ciudadano. En el marco de estas nuevas propuestas se subraya el planteamiento de que los estudiantes asuman el desarrollo sostenible como base del desarrollo humano, para tal fin se propone establecer la educación ambiental como eje transversal de los diferentes programas educativos, con el ánimo de fomentar la cultura ambiental en los ciudadanos. Dentro de estos planes se propone también que el tema energético se desarrolle conjuntamente dentro de los contenidos de las ciencias y además como proyecto transversal utilizando el enfoque de Ciencia, Tecnología y Sociedad (CTs) (Russel I, 2004).

Para el planteamiento de la educación energética desde el currículo escolar en los países latinoamericanos, se encuentra que cada país implementa una metodología diferente para la solución de esta problemática. Las diferentes naciones crean políticas educativas para la restructuración de currículos educativos, implementando medidas locales con características de problemática global, obedeciendo pautas generales aproximadamente iguales. Sin embargo la participación activa de la escuela no produce el impacto deseado, aún, para mejorar la cultura ciudadana en cuanto a su compromiso ambiental con el planeta, con el objetivo de mejorar la calidad de vida de las personas (Milachay. Y, 2005).
Dentro de las diversas propuestas metodologías orientadas hacia una educación energética de América latina es importante resaltar la implementada por Colombia en sus planes curriculares. Dentro de los cuales propone como objetivos generales para las ciencias naturales "formar en ciencias para contribuir a la formación de ciudadanos y ciudadanas capaces de razonar, debatir, producir, convivir, y desarrollar al máximo su potencial creativo. Esto es entendido como un compromiso para promover una educación ética, tolerante, con la diversidad y comprometida con el medio ambiente; una educación que se constituya en puente para crear comunidades con lazos de solidaridad, sentido de pertenencia y responsabilidad frente a lo público y lo nacional" (Ministerio Nacional de Educación de Colombia). La diferencia de los currículos comparados con otros, es que en lugar de presentar un listado de los contenidos temáticos se describe una lista de habilidades denominadas estándares con el objetivo que los estudiantes desarrollen a lo largo de su ciclo escolar habilidades para explorar, analizar, observar y recoger información, evaluar métodos y socializar información, es decir unos científicos en potencia. Estos estándares se articulan desde tres ejes básicos: los dos primeros pretenden desarrollar los contenidos formales, entorno vivo y entorno físico; mientras el tercero integra los aprendizajes previos con enfoque стs.

\section{Desarrollo sostenible}

En la última década, la preocupación por el calentamiento global que se ha plasmado en los diferentes acuerdos internacionales como Río de Janeiro, Kioto, Johannesburgo y Copenhague, que han dado paso a una visión amplia del estado de las ciudades, el medio ambiente, escasez de recursos y salud ecológica. Este cambio es parte esencial del desarrollo sostenible concepto que según Edwards (2008), enlaza dos grandes ejes de movimiento moderno la innovación tecnológica y la provisión social.

Como se ha mencionado anteriormente el proceso de construcción y estructuración del hábitat humano, ha ejercido un impacto sobre el medio natural 
hasta el punto de transformar radicalmente el territorio y en muchos casos agotar los recursos necesarios para la supervivencia, cuando sobrepasa el límite en que el suelo puede soportar en demanda, el sistema ecológico colapsa y por lo tanto se vuelve insostenible. En la medida en que este fenómeno se ha globalizado el término de desarrollo sostenible se ha convertido en un paradigma, ha generado discusión y hasta ha pasado a ser posición política (Cueto \& Villegas. 2007).

Bajo la búsqueda de estrategias de mitigación y disminución del impacto que se ocasiona al medio ambiente con las actividades relacionadas con edificaciones, surgen los tres principios básicos que nos llevan a un desarrollo sostenible, formulados por el economista Herman Daly citado por Alavedra, Domínguez, Gonzalo \& Serra (2000):

- Para una fuente de recursos renovable, no consumir a una velocidad superior a la de renovación natural.

- Para una fuente no renovable, no consumir sin dedicar parte necesaria de la energía resultante en desarrollar una "nueva fuente" que, agotada la primera, nos permita continuar disfrutando de las mismas prestaciones.

- Para un residuo, no generar más que aquel que el sumidero correspondiente sea capaz de absorber e inertizar de forma natural.

Según lo anterior y recordando que el objetivo principal de los edificios en un principio fue proteger a sus habitantes de los elementos naturales. Los principales esfuerzos se habían enfocado en este objetivo, actualmente el termino de desarrollo sostenible introdujo una restricción adicional que corresponde a cumplir su objetivo general sin comprometer la posibilidad de la generaciones futuras de satisfacer sus necesidades ${ }^{4}$, basados en los tres principios podrían ser una línea alternativa, para poder heredar a las generaciones futuras los recursos necesarios para su supervivencia.

4 Definición de desarrollo sostenible según la comisión Brundtland en 1987.

REVISTA CIENTÍFICA / ENERO -DICIEMBRE DE 2009 / No. 11 / BOGOTÁ, D.C.
Las primeras representaciones de construcción sostenible hacían hincapié en la dimensión energética y sobre todo en su diseño arquitectónico.

\section{Educación Energética}

La educación energética, para el ingeniero, enmarca toda una serie de aspectos bajo los cuales la formación académica y profesional de éste se desarrolla de manera integral, produciendo una construcción y uso del conocimiento a lo largo de todas sus etapas de acción tanto en el aula como posteriormente en su ejercicio profesional. Inicialmente la concepción de la energía como conocimiento científico genera una serie de procesos en los cuales el ingeniero en formación debe interactuar constantemente con este concepto en sus diferentes niveles de formación básica como mecánica, fluidos, termodinámica, electromagnetismo, las reacciones químicas, los procesos biológicos, entre otros. Se encuentra entonces que el concepto de energía está inmerso en todo el ciclo de formación de la ingeniería y su importancia radica en que es la energía la parte esencial para producir cualquier tipo de avance tecnológico y social en el mundo. Una de las problemáticas principales radica en la manera en cómo se aborda la enseñanza de este concepto donde, por ejemplo, en algunos casos se realiza la enseñanza de la energía fundamentalmente a través de su conservación y, en menor grado, por medio de su transformación, donde la transferencia y degradación son poco trabajadas. Estos esquemas fundamentales del concepto de energía se utilizan raramente para explicar fenómenos cotidianos, no abordables considerando únicamente la conservación (Solbes y Tarín, 1998).

Como segundo aspecto fundamental dentro de la educación energética, se encuentra la energía como componente de la educación ambiental; dentro de la cual se debe tener en cuenta que la educación científica se plantea ahora como parte de una educación general para toda la ciudadanía. Una educación que ha de prestar una mayor atención a aspectos como las relaciones cienciatecnología-sociedad, susceptibles de interesar a la 
mayoría de la población. (Doménech, Gil, Gras, Guisasola, Martínez, Salinas, Trumper, Valdés, 2003). Es aquí donde el ingeniero juega su papel más importante, dentro de su ejercicio profesional, con el aporte a la sociedad y a la preservación planetaria. Los ingenieros en cualquiera que sea su aporte al desarrollo tecnológico deben orientar sus trabajos a que tengan una visión macro de sostenibilidad; es decir, que deben funcionar como una sola armonía entre avance tecnológico y sostenibilidad del planeta. Es evidente notar el requerimiento esencial que tiene la formación en ingeniería del componente en educación energética, donde la concepción, estructura y manejo del concepto energético deben poseer una interrelación a lo largo de toda la formación del estudiante y su posterior aplicación profesional.

El tercer aspecto relevante dentro de la educación energética radica en la energía como componente de la formación laboral y práctica del alumno. Donde se encuentra que en gran medida los comportamientos de una persona están directamente ligados con la educación recibida en sus diferentes etapas de formación. El tipo de educación recibida, de acuerdo al sistema educativo, puede transformar las actitudes y modos de vida de un individuo modificando las concepciones e ideas de su contexto social y cultural. Paralelamente los comportamientos individuales de las personas están estrechamente ligados con su consumo energético, el cual depende de la disponibilidad de recursos, políticas locales, ingresos y precios de la energía (Educación Energética, 2006). La visión de un ingeniero debe encaminar los desarrollos industriales pero también aportar a los cambios procedimentales y de comportamiento de la sociedad, contribuyendo desde la ingeniería con metodologías y procesos que se inclinen por un desarrollo sostenible en todo su marco de avance social y científico.

\section{Metodología}

La problemática de las fuentes de energía se enmarca en una realidad que se caracteriza por ser compleja. Si bien las fuerzas sociales determi- nan que ésta es una necesidad factible de volverse un campo de investigación en la didáctica de las ciencias y que una de las formas de llegar a construir un conocimiento que oriente nuestras acciones es por medio de una comunidad global que actúe localmente y aporte globalmente. Además concebimos que existen personas capaces cognitivamente de comprender ésta problemática y de desarrollar soluciones, no podemos dejar de lado que una sola persona no lo puede hacer, ni lo puede comprender en totalidad, y que una sola acción, teoría, un solo desarrollo procedimental inclusive una sola forma de valorar la problemática no es ni será suficiente. En otras palabras esta realidad es compleja porque: no encierra la compresión de un único fenómeno, las teorías que lo podrían explicar incluye un conjunto de conceptos diversos y en constante cambio, una sola disciplina no puede cubrir la totalidad del problema y finalmente porque el estudio no es sobre un objeto en particular es sobre un sistema ecológico no lineal, un ecosistema terrestre.

\section{Estrategia Metodológica}

Este proyecto de investigación se enmarca dentro de la denominada investigación alternativa, donde se renuncia a la dicotomía entre métodos cualitativos y cuantitativos, ya que se concibe que un dato cuantitativo se construye con base en juicios cualitativos y todo dato cualitativo es posible de cuantificar, además que la validez del conocimiento construido se logra de mejor manera cuando los dos tipos de datos se complementan (Páramo y Otálvaro, 2006).

La presente investigación se ha desarrollado bajo la estrategia de investigación llamada teoría de facetas puesto que ésta permite integrar el dominio de interés de la investigación, en este caso son las estrategias de enseñanza sobre el concepto de energía que favorezca el desarrollo sostenible y sustentable, con el análisis de la información que se pueda recolectar en los planes de estudio, los libros más consultados, y lo que opinan los profesores de física para ingenieros sobre las estrategias de enseñanza y sobre el concepto de energía, 
ya que ofrece herramientas y procedimientos para analizar, estructurar y reestructurar los contenidos de investigación (Páramo, 1999).

La teoría de facetas es una metateoría, es decir una teoría de la teoría, que plantea una metodología que permite integrar tres componentes de la investigación: una hipótesis de correspondencia entre un sistema definitorio para un universo de observaciones, un aspecto de la estructura empírica de dichas observaciones y una racionalidad para tal hipótesis (Páramo, 1999).

Además, se considera que la teoría de facetas es una estrategia adecuada para este tipo de investigación en tanto que permite definir tres tipos de facetas: una de contexto, otra de dominio y otra de rango. Con la primera, las facetas de contexto, permite describir el contexto del estudio, que para nuestro caso vendría siendo procesos de formación de ingenieros desde el departamento de ciencias básicas de la Fundación Universitaria Los Libertadores. La segunda, las facetas de dominio, describe el cuerpo de área de interés, en este caso serían las dificultades y recomendaciones que hacen posible mejorar los procesos de enseñanza y aprendizaje del concepto de energía. La tercera, la facetas de rango, las cuales permiten describir las posibles respuestas que emergen de los estímulos suministrados por las facetas de dominio, por ejemplo, en el caso que nos ocupa, frente a la afirmación "el calor no es un tipo de energía" se espera que los profesores de física estén en total acuerdo con dicha afirmación.

\section{Conclusiones}

Esperamos que dar a conocer este proyecto de investigación permita visibilizar la importancia de la educación científica de los ingenieros para poder alcanzar una educación energética y en términos generales una educación para la sostenibilidad que le haga frente a problemas como el energético. Además, que podamos seguir construyendo comunidad de investigadores en didáctica de las ciencias que abordan este tipo de problemas.

\section{Bibliografía}

Alcantud, J. L. (2007). La atención a la situación del mundo en el tratamiento de la energía realizado por la educación tecnológica. Tesis Doctoral. Universitat de València.

Arias, A. G. (2006). El concepto "energía” en la enseñanza de las ciencias. En: Revista Iberoamericana de Education. 38, (2).

Arnold, M. (1994). Children's and lay adults' views about thermal equilibrium. En: International Journal of Science Education, 16(4): 405-419.

Brown, L. (2008). Plan B 2.0 Rescatando un planeta bajo estrés y una civilización en dificultades. Bogotá: Editorial Universidad del Rosario.

Carmona, A. (2009). Investigación en didáctica de la Física: tendencias actuales e incidencia en la formación del profesorado. En: Latino American Journal Physics Education. 3(2).

Carr, M. \& Kirkwood, V. (1988). Teaching and learning about energy in New Zealand secondary school junior science classrooms. En: Physis Education. 23: 87-91.

Cuelto, G. y Villegas, J. (2007). ¿Es la arquitectura responsable del calentamiento global?. En: Revista Javeriana. Julio: 22-29.

Organización de los estados Iberoamericanos OEI (2002) Proclamación de la Década de las Naciones Unidas de la Educación para el Desarrollo Sostenible. Resolución 57/254 aprobada por la Asamblea General de las Naciones Unidas el 20 de Diciembre Acceso en 23 de febrero de 2010 Disponible en: http://www. oei.es/decada/resonu.htm

Doménech, J. L. L., Gil, D., Gras, A., Guisasola, J., Martínez, J., Salinas, J., Trumper, R., Valdés, P. (2003). La Enseñanza de la Energía: una Propuesta de debate para un replanteamiento global. En: Caderno Brasilera de Enseñanza de la Física. 20 (3): 285-310. 
Driver, R. y Warrington, L. (1985) Students' use of the principle of energy conservation in problem situations. Physics Education, 20, pp. 171-176.

Duit, R. (1981) Understanding Energy as Conserved Quantity-Remarks on the Article by R.U. Sexl. European Journal of Science Education, 3(3), pp. 291-31..

Duit, R. (1981) Understanding Energy as Conserved Quantity-Remarks on the Article by R.U. Sexl. European Journal of Science Education, 3(3), pp. 291-301.

Duit, R. (1984)Learning the energy concept in school empirical results from The Philippines and West Germany. Physics Education, 19, pp. 59-66.

Duit, R. (1987) Should energy be illustrated as something quasi-material? European Journal of Science Education, 9(2), pp. 139-145.

Dussel, I. (2004.) Las políticas generales de la última década en América Latina: nuevos actores, nuevos problemas, FLACSO, Buenos Aires,

Comunidad Europea (2006) Educación Energética. Enseñar a los futuros consumidores de energía. Comisión Europea. Luxemburgo: Oficina de Publicaciones Oficiales de las Comunidades Europeas.

Edward B. (2008) Guía de sostenibilidad. Editorial Gustavo Gili. Barcelona. España. Págs. 223.

Erickson, G. (1979) Children's conceptions of heat and temperature. Science Education, 63(2), pp. 221-230.

Flannery, T. (2006) El clima está en nuestras manos. Historia del Calentamiento Global. Editorial Taurus.

Guisasola, J., Gras-Marti, A., Martinez-Torregosa, J. Almundi, J., Becerra, C. (2004) La Ense- ñanza Universitaria de la Física Y Las Aportaciones de la Investigación en Didáctica de la Física. Revista Española de Física 18, 15-16 (2004).

Lovelock, J. (2004) La venganza de la tierra. La teoría Gaia y el futuro de la humanidad

Páramo, P. y Otalvaro, G. (2006) Investigación alternativa: Por una distinción entre posturas epistemológicas y no entre métodos. En: Cinta de Moebio 25. Universidad de Chile. www. moebio.uchile.cl/25páramo.html (Última Visita: Septiembre 21 de 2008)

Páramo, P. (1999) Teoría de Facetas: Guía Metodológica para la Recolección de Opiniones dentro del Proceso de Autoevaluación Institucional. En: Revista Saberes. Universidad Pedagógica Nacional.

Pernick Ron \& Wilder Clint. (2000) La revolución Limpia. Invertir en tecnología y crecer en el futuro inmediato. Editorial Gestión

Sexl, R.U. Some Observations Concerning the Teaching of the Energy Concept. European Journal of Science Education, 3(3), pp. 285-289. 1981.

Milichay, Y., Gras-Marti, A., y Cano-Villalba, M. (2005) La educación energética en América Latina. En: Educación, Energía e Desenvolvemento Sostenible, pp. $387-406$

Solbes. J y Tarín. F. (1998) Algunas dificultades en torno a la conservación de la energía. Ensenanza de las ciencias: revista de investigación y experiencias didácticas, Vol. 16, No. 3, p. 387-398.

Solomon, J. (1985) Teaching the conservation of energy. Physics Education, 20, pp. 165-176...

Solbes, J. y Martin, J. (1991) Análisis de la introducción del concepto de campo. Revista Española de Física, 5, pp. 34-40... 
Van Huls, C. y Van Den Berg. (1993) Teaching energy: a systems approach. Physics Education, 28, pp. 146-153.

Von Roon, P.H., Van Sprang, H.F. y Verdonk, A.H. (1994) Work and Heat: on a road towards thermodynamics. International Journal of Science Education, 16(2), pp. 131-144.
Warren, J.W. (1982) The nature of energy. European Journal of Science Education, 4(3), pp. 295-297.

Weart, S. (2006) El calentamiento global. Historia de un descubrimiento científico. Laetoli. Harvard University Press, 2003. 